Received: 21 February 2017

Accepted: 17 May 2017

Published online: 27 June 2017

\section{Danhong injection reduces vascular remodeling and up-regulates the Kallikrein-kinin system in spontaneously hypertensive rats}

Xiaohu Yang ${ }^{1,2,6}$, John Orgah ${ }^{1,2}$, Dandan Wang ${ }^{1,2}$, Guanwei Fan ${ }^{1,2}$, Hu Jingyang ${ }^{1,2}$, Jihong Han $^{3}$, Gangjian Qin ${ }^{1,2,4}$, Xiumei Gao ${ }^{1}$ \& Yan Zhu ${ }^{1,2,5}$

Although Danhong injection (DHI) is one of the most prescribed cardiovascular medicines in China, its therapeutic indications and mechanisms remain partially defined. We now identify molecular targets of $\mathrm{DHI}$ in resistance vasculatures and demonstrate its role in vascular function and blood pressure (BP) regulation. BP was determined in DHI, Losartan, and placebo- treated Spontaneously Hypertensive Rats (SHR) by both noninvasive and invasive measurements. Vasorelaxation was examined both in conduit and resistance vasculature by ex vivo aortic rings. Microarray analysis was performed and gene expression changes were verified by RT-qPCR and ELISA. Diastolic, systolic and mean BPs were significantly lower in DHI-treated SHR than controls by both tail-cuff and invasive BP measurements. In ex vivo rings, aortic and mesenteric vessels from SHR treated with DHI exhibited significantly greater acetylcholine-mediated relaxation. Among the $\mathbf{2 8 2}$ genes that are differentially expressed in microarray analysis, DHI treatment up-regulated the expression of kallikrein and plasma kallikrein B genes. DHI also significantly increased serum kallikrein content in SHR. Treatment with DHI significantly increased the ratio of aortic lumen to outer diameter. Therefore, the reduction of vascular remodeling and the upregulation of Kallikrein-kinin system contribute, at least in part, to the antihypertensive effect of DHI in SHR.

Hypertension is a major public health issue and a leading cause of premature death in China ${ }^{1}$. Approximately 1 billion people worldwide have high blood pressure (BP), and recent results of a large prospective cohort study of Chinese population suggest that out of the 500,223 people recruited, there were approximately 162,572 people with hypertension ${ }^{2}$. That translates to one in three adults who suffer from high BP. Despite over $\$ 19$ billion in worldwide sales of antihypertensive agents, a considerable number of patients remain resistant to treatment. Novel treatment strategies are required to address this unmet medical need. As a major cardio-cerebrovascular risk factor, hypertension causes constriction of the microvasculature, resulting in the dysfunction of target organs such as the heart, brain and kidney ${ }^{3}$. Because the microcirculation provides the vast majority of systemic resistance to flow, as well as virtually all the oxygen and nutrient exchange ${ }^{4}$, alterations in microcirculatory function and structure are of great interest in the syndrome of hypertension and its target organ consequence ${ }^{5,6}$. Microvascular abnormalities during hypertension increase vascular sensitivity, vessel wall thickness and loss of capillaries ${ }^{7}$.

The etiology of elevated BP cannot be determined in the vast majority of individuals with essential hypertension and many systems have been shown to contribute to BP homeostasis, including the vasculature, the central and sympathetic nervous systems, and the kidney, along with their various hormonal regulators. Multiple

${ }^{1}$ Tianjin State Key Laboratory of Modern Chinese Medicine, Tianjin University of Traditional Chinese Medicine, 312 Anshanxi Road, Nankai District, Tianjin, 300193, P. R. China. ${ }^{2}$ Research and Development Center of TCM, Tianjin International Joint Academy of Biotechnology \& Medicine, 220 Dongting Road, TEDA, Tianjin, 300457, P. R. China. ${ }^{3}$ College of Life Sciences, Nankai University, Tianjin, 300193, P. R. China. ${ }^{4}$ Feinberg Cardiovascular Research Institute, Department of Medicine, Northwestern University Feinberg School of Medicine, Chicago, IL, USA. ${ }^{5}$ Molecular Cardiology Research Institute, Tufts Medical Center, 750 Washington St, Boston, MA, 02111, USA. ${ }^{6}$ Present address: Department of Pharmacy, Zhejiang Hospital, 12 Lingyin Road, Xihu District, Hangzhou, Zhejiang, 310013, P. R. China. Correspondence and requests for materials should be addressed toY.Z. (email: yanzhu.harvard@icloud.com) 
pathways such as the renin-angiotensin aldosterone system (RAAS), NO/cGMP ${ }^{8,9}$ and Kallikrein-kinin system (KKS) are considered as the major BP-regulating mechanisms ${ }^{10-12}$. Normal levels of kallikrein are required in the maintenance of BP since tissue kallikrein levels are reduced in humans and/or in animal models with hypertension $^{11,13}$. Genetic models of kallikrein deficiency further support the role of kallikrein in BP regulation ${ }^{14}$. Additionally, up-regulation of kallikrein also has a critical role in vascular protection, especially in prophylaxis of vascular smooth muscle cell (VSMC) proliferation, vascular wall thickness and vascular remodeling ${ }^{15-19}$.

Quite a large number of clinical evidence suggested that multiple drug combinations produce BP reductions superior to single drug use and may help difficult-to-treat patients to achieve BP control ${ }^{20}$. Accordingly, herbal medicine, a natural multi-component medicine, has long been used in treating cardiovascular disease such as hypertension ${ }^{8}$. Although many herbal-derived antihypertensive medicines are shown to be safe and effective, complex chemical nature have limited in-depth investigation of their action mechanisms $s^{8,21,22}$.

Microarray technology has been successfully applied to elucidate the mechanism associated with complex therapeutic effects of herbal medicine ${ }^{23}$. Analyzing the alterations of gene expression profiles after treatment by herbal medicine in vitro/vivo may help elucidate their mechanisms of action ${ }^{24,25}$.

Danhong injection (DHI), a traditional Chinese Materia Medica standardized product extracted from Radix Salviae miltiorrhizae (Danshen) and Flos Carthami tinctorii (Honghua) ${ }^{26}$, was approved by State Food and Drug Administration of China (Permission Number Z20026866). It was formulated based on the traditional Chinese medicine (TCM) theory of "activating and promoting blood circulations to dissipate blood stasis and dredge collaterals" 27

To our knowledge, DHI is among the best chemically characterized complex herbal medicine. We have previously characterized the major constituents of DHI by ultra-performance liquid chromatography (UPLC) ${ }^{28}$ and proton nuclear magnetic resonance $\left({ }^{1} \mathrm{H} \text { NMR }\right)^{29}$. Recently, Zhang et al. have separated and identified further constituents in DHI by UPLC ${ }^{30}$.

DHI has been widely used in Chinese hospitals and clinics with proven efficacy and safety ${ }^{31}$ for the treatment and prophylaxis of various cardio-cerebrovascular diseases, such as coronary heart disease, atherosclerosis, and ischemia-reperfusion injury ${ }^{32-34}$. Recently published evidence suggests that DHI exert anti-cardiac hypertrophic effect by regulating $\mathrm{p} 38$ and NF- $\kappa \mathrm{b}$ pathway ${ }^{35}$, ameliorates cardiac dysfunction and ventricular remodeling after myocardial infarction ${ }^{36}$, and relaxed norepinephrine-induced vasoconstriction via inhibition of the intracellular calcium ions $\left(\mathrm{Ca}^{2+}\right)$ or via a $\mathrm{PGI}_{2}$-mediated pathway ${ }^{37,38}$. However, its role on $\mathrm{BP}$ regulation and vascular protection has not been explored.

The present study aimed to investigate (1) the potential role of DHI on BP regulation and vascular remodeling in spontaneous hypertensive rat (SHR), (2) the ability of DHI in restoring endothelial dysfunction in both conduit (aortic) and resistance (mesenteric) vessels of SHR rats, and (3) the underlying mechanisms and signaling pathways of the microvascular activities by DHI.

\section{Results}

DHI Lowered Blood Pressure in SHR. We first evaluated the effect of DHI on BP regulation in SHR and then compared the result with that of negative control group (SHR + Saline), taking Losartan as positive control drug. As expected, Losartan dramatically lowered diastolic, systolic and mean BP in SHR starting from day 1 till after 4 weeks (from $148.7 \pm 4.2$ to $107.3 \pm 10.1 \mathrm{mmHg}, \mathrm{p}<0.05 ; 194.3 \pm 8.9$ to $144.7 \pm 9.9 \mathrm{mmHg}, \mathrm{p}<0.05$; and $163.8 \pm 8.3$ to $119.4 \pm 9.9 \mathrm{mmHg}, \mathrm{p}<0.05$, respectively. Supplemental Materials Figure $\mathrm{S} 1 \mathrm{~A}-\mathrm{C}, \mathrm{n}=4)$. Although treatment with DHI had no such immediate and aggressive effect on BP reduction in SHR as observed with Losartan, the diastolic, systolic and mean BP were all significantly decreased compared to those of saline controls within 7 days till 4 weeks (from $147.9 \pm 7.9$ to $127.7 \pm 4.9 \mathrm{mmHg}, 194.3 \pm 8.9$ to $177.3 \pm 2.2 \mathrm{mmHg}, 163.8 \pm 8.3$ to $143.8 \pm 6.9 \mathrm{mmHg}$, respectively, $\mathrm{p}<0.05, \mathrm{n}=4$, Fig. $1 \mathrm{~A}-\mathrm{C}$ ). This mild BP lowering effect of DHI was apparently less traumatic since it did not cause a significant weight loss ( $p>0.05$ vs. saline group, Fig. 1D), and possibly avoided detrimental organ damage in rats caused by acute reduction of BP using a BP lowering drug as previously reported ${ }^{39}$.

In addition to noninvasive $\mathrm{BP}$ records, invasive $\mathrm{BP}$ monitoring was performed in anaesthetized rats which confirmed that DHI smoothly and effectively decreased $\mathrm{BP}(\mathrm{p}<0.01, \mathrm{n}=3$, Fig. 2A, top panel [SHR (DHI)] and $2 \mathrm{~B})$ but did not change the heart rate ( $\mathrm{p}>0.05$, Fig. $2 \mathrm{C}$ ) in SHR. Moreover, DHI did not change BP in normotensive WKY rats (Fig. 2A, lower panel [WKY DHI]).

DHI Improved Endothelial Dysfunction in SHR. Since we have previously reported that DHI enhanced acute vasorelaxation in wild-type Sprague Dawley (SD) rats $^{38}$, we conducted the same ex vivo vascular ring assay using thoracic aortas isolated from SHR. After one week of DHI treatment, percentage relaxation rate (\%) of thoracic aortas in response to Ach $\left(1 \times 10^{-8}\right.$ to $1 \times 10^{-6} \mathrm{~mol} / \mathrm{L}$ ) was significantly improved (from $0.16 \pm 0.054$ to $0.39 \pm 0.029, \mathrm{p}<0.01, \mathrm{n}=5$, Fig. $3 \mathrm{~A}$ and $\mathrm{C}$ ) whereas relaxation rate (\%) in response to sodium nitroprusside $(\mathrm{SNP})$ remained the same $\left(1 \times 10^{-9}\right.$ to $1 \times 10^{-7} \mathrm{~mol} / \mathrm{L}, \mathrm{p}>0.05, \mathrm{n}=5$, Fig. $3 \mathrm{~B}$ and $\left.\mathrm{D}\right)$. As expected, SHR were deficient in endothelium-dependent vasorelaxation ${ }^{40-42}$. Because resistance vessels (mesenteric vessels) are more critical for BP control, we then conducted further vasorelaxation assay using mesenteric vessels isolated from DHI-treated SHR. After three days of DHI treatment, mesenteric arteries in SHR showed an enhanced relaxation (from $0.44 \pm 0.12$ to $0.58 \pm 0.05, \mathrm{p}<0.01, \mathrm{n}=3$ ) in response to Ach $\left(1 \times 10^{-11}\right.$ to $1 \times 10^{-9} \mathrm{~mol} / \mathrm{L}$, Fig. $4 \mathrm{~A}$ and $\mathrm{C}$ ) compared to controls (Fig. 4B and C). Finally, DHI caused a direct dose-dependent relaxation of isolated mesenteric vessels (Fig. 4D and F) compared to the controls (Fig. 4E and F).

DHI Reversed Vascular Remodeling in SHR. Aortic thickness resulted in the reduction of their lumens. Compared with that of WKY rats, the aortas from SHR showed a significant decrease in the ratio of lumen/outer diameter (from $0.904 \pm 0.007$ to $0.842 \pm 0.003, \mathrm{p}<0.05, \mathrm{n}=4$, Fig. $5 \mathrm{~A}, \mathrm{~B}$ and $\mathrm{E}$ ). As expected, Losartan increased 
A

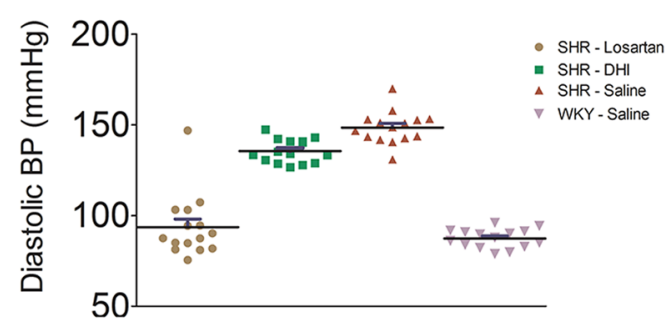

B

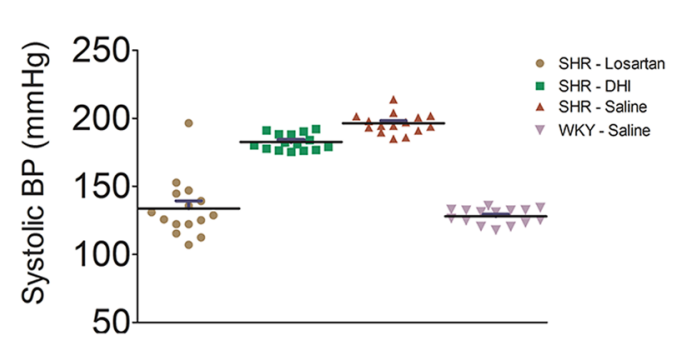

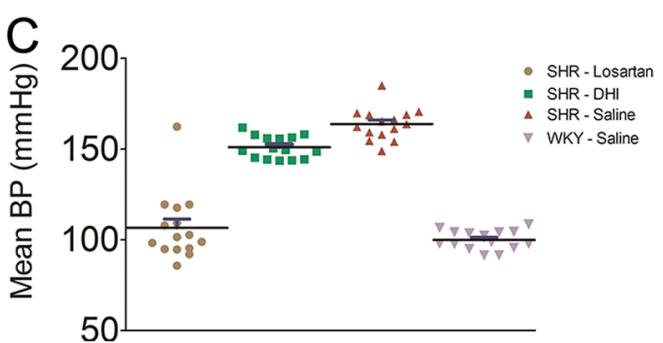

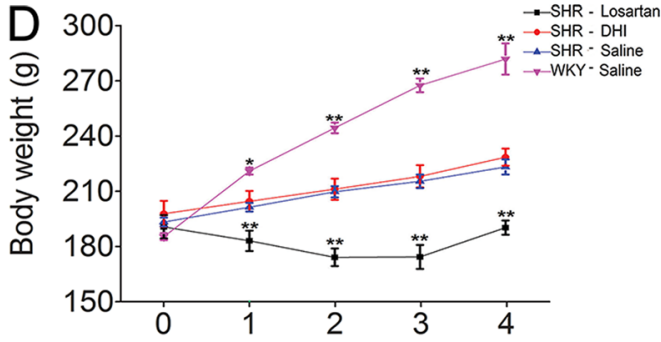

Figure 1. Effects of DHI on noninvasive blood pressure in SHR. Panel A-C are representative data plots from (DBP, SBP, and MBP measurements, respectively) using noninvasive tail-cuff method in conscious rats. Panel D represent body weight in (gram) measured with the small animal weighing scale. Compared with saline control, DHI-treated SHR showed no significant difference ( $p>0.05$ vs. saline group) in weight over the 4 weeks of routine treatment. Data are expressed as mean \pm SEM, $\mathrm{n}=4$.

A
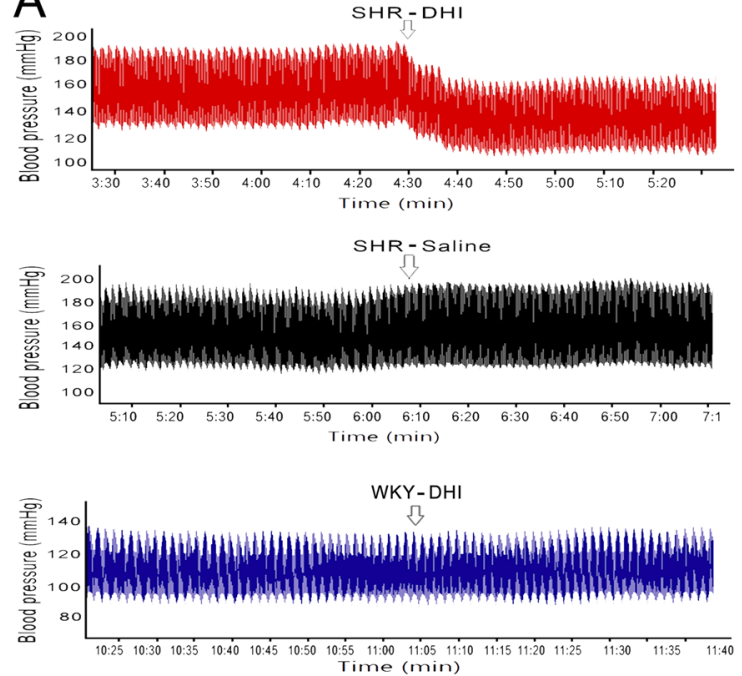
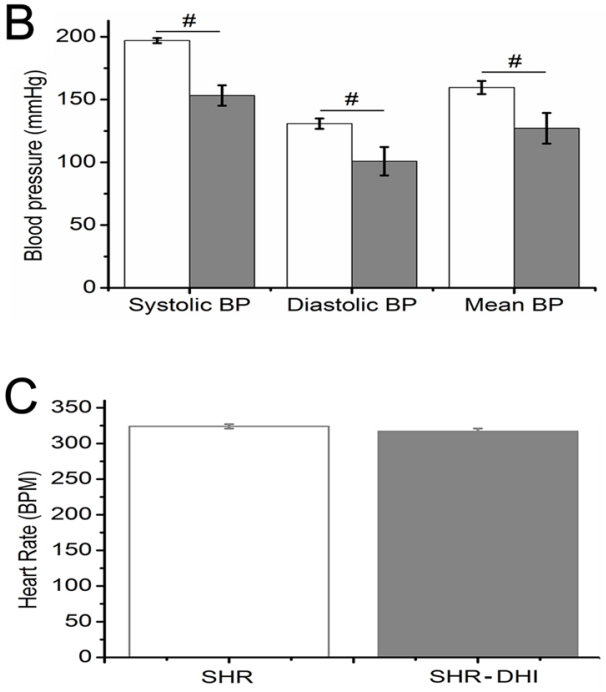

Figure 2. Effects of DHI on invasive blood pressure in SHR. Invasive BP were measured in urethane anaesthetised rats after treatment with DHI. A heparinized saline, $100 \mathrm{IU} / \mathrm{mL}$ was filled in the transducer in clean polyethylene catheter cannulated to the ventricle through the left common carotid artery. DHI decreased BP in SHR ( $<<0.01$, Fig. 2A [SHR (DHI)] and $2 B, n=3)$ but did not change the heart rate $(p>0.05$, Fig. 2C). DHI did not change BP in normotensive WKY rats ( $\mathrm{p}>0.05$, Fig. 2A [WKY (Saline)]).

the ratio of lumen/outer diameter (Fig. 5B,D and E). Similarly, DHI treatment significantly increased the ratio of lumen/outer diameter (from $0.842 \pm 0.003$ to $0.891 \pm 0.004, \mathrm{p}<0.05, \mathrm{n}=4$, Fig. $5 \mathrm{~B}, \mathrm{C}$ and $\mathrm{E}$ ). The VSMC layer of DHI-treated SHR was thinner than that of their control counterpart (from $62.4 \pm 0.9$ to $47.8 \pm 0.6 \mu \mathrm{m}, \mathrm{p}<0.05$, $\mathrm{n}=4$, Fig. 5B [x400], 5C [x400] and 5F).

Identification of Differentially Expressed Genes from Microarray and RT-PCR validation. In order to identify the molecular mechanisms of DHI effect on vascular relaxation and remodeling, we compared gene expression profiling between DHI-treated and control-treated SHR using the Rat Genome-230 2.0 microarray (31,000 genes represented). Mesenteric vessels from four independent animals per group were individually analyzed (Supplemental Figure S2A) and the results indicates that a total of 282 genes (195 up-regulation and 87 down-regulation, Supplemental Figure S2B and C, details in Supplemental Materials Table S1) were altered when a cutoff of t-test $(\mathrm{p}<0.05)$ and fold changes $(>2)$ was applied. 
A

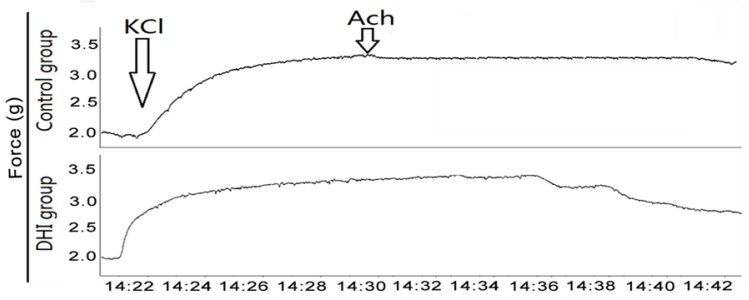

B

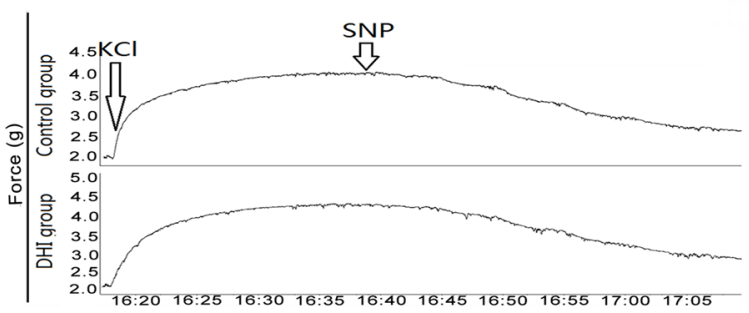

C

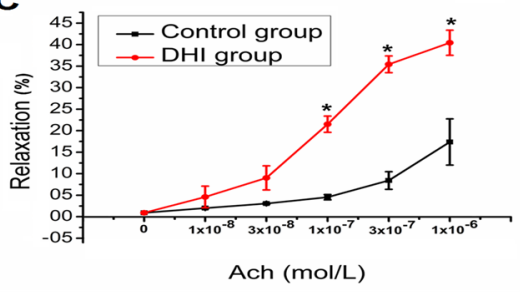

D

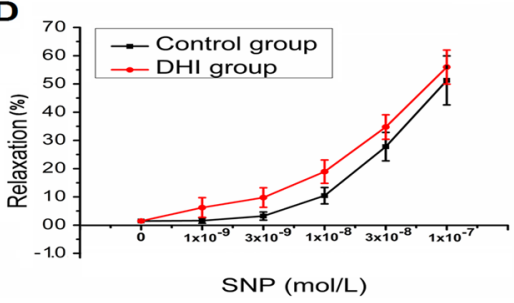

Figure 3. Ach and SNP-mediated vasorelaxation in $\mathrm{KCl}$ - precontracted thoracic aortas isolated from DHItreated SHR. (A,B) Raw trace of Ach and SNP-treated aorta and $(\mathbf{C}, \mathbf{D})$ Quantification of the data in $(\mathbf{A}$ and $\mathbf{B})$ respectively. The rate of Ach and SNP-induced relaxation was calculated with $\mathrm{KCl}(60 \mathrm{mM})$-induced contraction set to 0 . (C) DHI treatment significantly $(\mathrm{p}<0.01)$ relaxed aorta in response to Ach stimulation compared with control group in SHR. (D) There was no significant difference in response to SNP stimulation between DHI treatment group and control group in SHRs' aorta. All data are expressed as mean \pm SEM, $n=5$.

The microarray data was validated as Losartan significantly down-regulated angiotensin/aldosterone genes in SHR (Fig. 6A). However, DHI did not alter these angiotensin/aldosterone genes (Fig. 6B), nor the NOS genes (Fig. 6C) in SHR. On the other hand, DHI did alter several genes known to be associated with BP-regulation and hypertension, including kallikrein, plasma kallikrein B1 (Klkb1), 5-hydroxytryptamine (serotonin) receptor 6 (Htr6) and potassium inwardly-rectifying channel, subfamily J, member 2 (Kcnj2) and their differential expressions were validated by RT-PCR from the same RNA source (Fig. 6F). Of particular interest, the expression of kallikrein and Klkb1 genes were up-regulated in microarray by $2.19 \pm 0.12$-fold change and $2.11 \pm 0.16$-fold change respectively $(\mathrm{p}<0.05, \mathrm{n}=4$, Fig. $6 \mathrm{~F})$ which were confirmed by RT-PCR by $2.23 \pm 0.57$-fold change and $2.88 \pm 1.01$-fold change, respectively $(\mathrm{p}<0.05, \mathrm{n}=4$, Fig. $6 \mathrm{~F})$. As a control, von Willebrand factor (vWF), an unaltered gene in microarray, was also not altered in RT-PCR (data not shown).

DHI Selectively Increased Serum Kallikrein Content in SHR. To further distinguish the BP-regulation pathways affected by DHI, we conducted ELISA assays for the relevant factors. While DHI did not alter the levels of renin, angiotensin and aldosterone contents $(\mathrm{p}>0.05, \mathrm{n}=4$, Fig. 6D), it significantly increased serum kallikrein content (from $3.15 \pm 0.1$ to $3.80 \pm 0.2 \mathrm{ng} / \mathrm{mL}, \mathrm{p}<0.05, \mathrm{n}=4$, Fig. $6 \mathrm{E}$ ) in SHR compared with the controls.

\section{Discussion}

Formulated based on the TCM theory of "activating and promoting blood circulations to dissipate blood stasis and dredge collaterals" ${ }^{\prime 3}$, DHI has been widely used in Chinese hospitals and clinics with proven efficacy and safety ${ }^{31}$ for treatment and prophylaxis of various cardio-cerebrovascular incidents, such as treatment of ischemia-reperfusion injury, atherosclerosis, acute coronary syndrome, hepatic veno-occlusive disease ${ }^{32,33,43,44}$. Following our previous published work ${ }^{38}$ showing that DHI and one of its component Danshensu serve as a direct vasodilator in normal rats, the primary finding of this study is that DHI significantly lowered BP (diastolic, systolic and mean blood pressure) in SHR compared with that of control group (Fig. 1A-C). It is worth noting that while its BP-lowering effect was not as robust as Losartan, DHI did not cause adverse effect resulting in the rapid weight loss as the Losartan treatment group (Fig. 1D). Consistent with this observation, we also found that the level of myoglobin $(\mathrm{Mb})$, a biomarker for kidney injury, was much higher in Losartan group compared to those of DHI and control groups (data not shown). The milder and smoother effect of DHI in lowering BP compared with Losartan (Fig. 1A-C) indicated that DHI may avoid some of the known adverse effects of antihypertensive drugs ${ }^{8,39,45}$. Furthermore, the dual effects of direct vasorelaxation and long-term vascular remodeling by DHI support the effectiveness of multi-targeting strategy in blood pressure control, an advantage of Traditional Chinese Medicines with multicomponent combinations.

DHI is formulated as an injection using aqueous extracts from Radix Salviae miltiorrhizae (Danshen) and Flos Carthami tinctorii (Honghua) and has been shown significantly to vasodilate isolated arterial rings in rats ${ }^{38}$. Several studies have indicated that various extracts and components from either Danshen or Honghua may have anti-hypertension activities ${ }^{46-48}$. Pei-He Nie et al. ${ }^{46}$ and David D. Kim et al. ${ }^{48}$ reported that HSYA (a hydrophilic compound from Honghua) and tanshinone IIa (a lipophilic compound from Danshen) had an antihypertensive effect. However, our recent chemical composition analysis of $\mathrm{DHI}^{28}$ showed that neither HSYA nor tanshinone IIa were detectable. Therefore, the antihypertensive effect of DHI in our study could not be attributable to HSYA and tanshinone IIa. Other studies also found that antihypertensive effects of Danshen and Danshensu (a water-soluble 
A

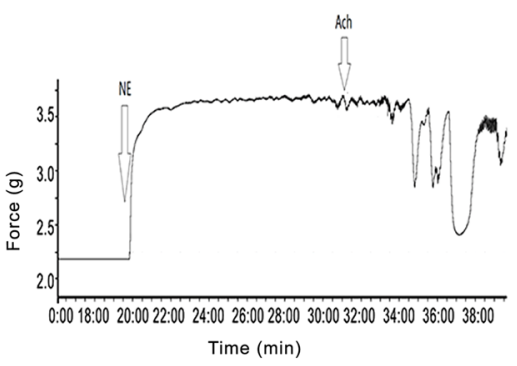

D

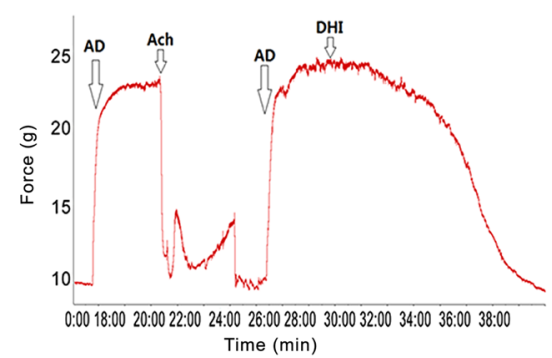

B

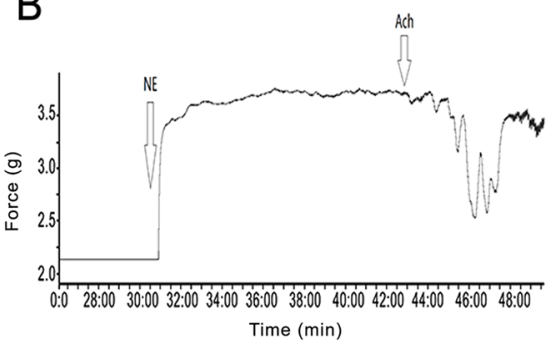

$\mathrm{E}_{22}$

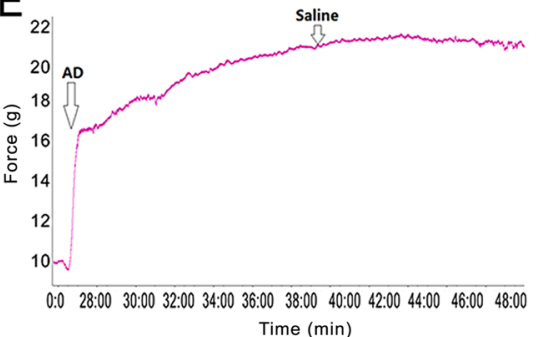

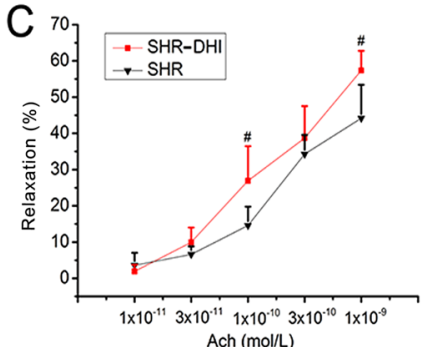

$\mathrm{F}$

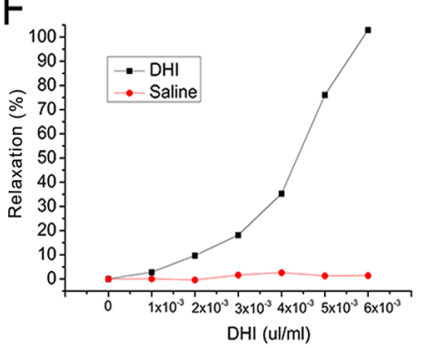

Figure 4. In vivo and ex vivo effects of DHI on mesenteric arteries from SHR and wild-type rats. (A,B) Raw traces of relaxation of NE-precontracted MAs from SHR in response to ACh (DHI vs. Saline treatment). (C) Quantitation of data in A and B expressed as \% relaxation in response to increased Ach concentrations. (D,E) Raw traces of relaxation in response to ACh (DHI vs. Saline treatment) on AD pre-contracted mesenteric arteries. (F) Quantitation of data in (D and $\mathbf{E})$ expressed as \% relaxation in response to increased DHI concentrations. The rate of Ach or DHI-induced relaxation was calculated with NE (10 mM) or AD $(10 \mathrm{mM})$ contraction set as 100\%. (A and B) Mesenteric arteries from DHI-treated SHR animals for three days significantly relaxed more than that of saline control in response to increasing doses of ACh, $\mathrm{p}<0.01$. (D and E) DHI treatment also caused a direct endothelial-dependent vasorelaxation in $\mathrm{AD}$ pre-contracted mesenteric arteries from normal $(\mathrm{WKY})$ rats, $\mathrm{p}<0.01$. All data are expressed as mean $\pm \mathrm{SEM}, \mathrm{n}=3$.

compound from Danshen) were mediated by the inhibition of angiotensin conversion enzyme (ACE) and the activation of NOS/NO, respectively ${ }^{9,47}$. Although our previous study showed that Danshensu was a major component of DHI involved in endothelium-dependent vasorelaxation in wild-type SD rats ${ }^{38}$, our current microarray analyses of resistant vessels from SHR demonstrated that ACE and NOS gene expression were not altered by DHI (Fig. 6B and C). These findings provided a clear distinction of antihypertensive effects of DHI from isolated compounds and extracts of Danshen and Honghua reported previously.

Since DHI is an herbal extract-derived medicine, its antihypertensive effect could be contributed by multiple components through a variety of mechanisms. Therefore, we performed a microarray analysis of the genes in resistance vessels to discover the potential genes regulated by DHI and to investigate the mechanistic insights of its BP-lowering effects. The microarray data for 8 samples were firstly analyzed by hierarchical clustering analysis for a quality evaluation and a comparison of the treatment effects. The biological replicates in each treatment group showed high reproducibility judged by heat-map analysis (Supplemental Figure S2A). Our results indicate that in micro-vessels of SHR, DHI altered multiple pathways such as insulin signaling pathway, glutathione, mTOR signaling pathway (See details in Supplemental Materials Table S3).

Microarray data analyses indicated that in contrast to that of Losartan (Fig. 6A), DHI did not alter the expression of genes such as renin, angiotensin and aldosterone (RAAS, Fig. 6B), suggesting that the DHI pathway for reducing $\mathrm{BP}$ was not mediated by RAAS. On the other hand, we found several differentially expressed genes that were related to BP regulation, especially kallikrein gene that was significantly overexpressed (Fig. 6F).

RAAS and KKS are two major circulating endocrine systems in the regulation of $\mathrm{BP}^{10,49}$. Cross-talks exist between the two systems and their levels of expression showed a complex pattern: both RAAS and KKS would be up-regulated in some circumstances, whereas in other circumstances they respond to opposite direction, expressed as an activated KKS and a depressed RAAS ${ }^{50}$. As the cross-talks between the RAAS and KKS play a critical role in regulation of $\mathrm{BP}^{51}$, we further investigated if $\mathrm{DHI}$ affected one or both of the pathways. Indeed, our ELISA tests of serum renin, angiotensin, aldosterone and kallikrein (Fig. 6D and E) were in excellent accord with the gene expression data (Fig. 6B and F). As a main component of KKS, Kallikrein plays a crucial role in regulation of $\mathrm{BP}$ and thus a target for antihypertensive drugs ${ }^{14,52,53}$. In our study, results of ELISA analysis and microarray data indicated consistency with several recent studies ${ }^{14,54}$, kallikrein gene overexpression in mesenteric micro-vessels increased the serum kallikrein content. Therefore, our results seem to support a differential pattern of RAAS and KKS by DHI and the BP-lowering effect of DHI is preferentially triggered by KKS mediated by kallikrein gene overexpression. 

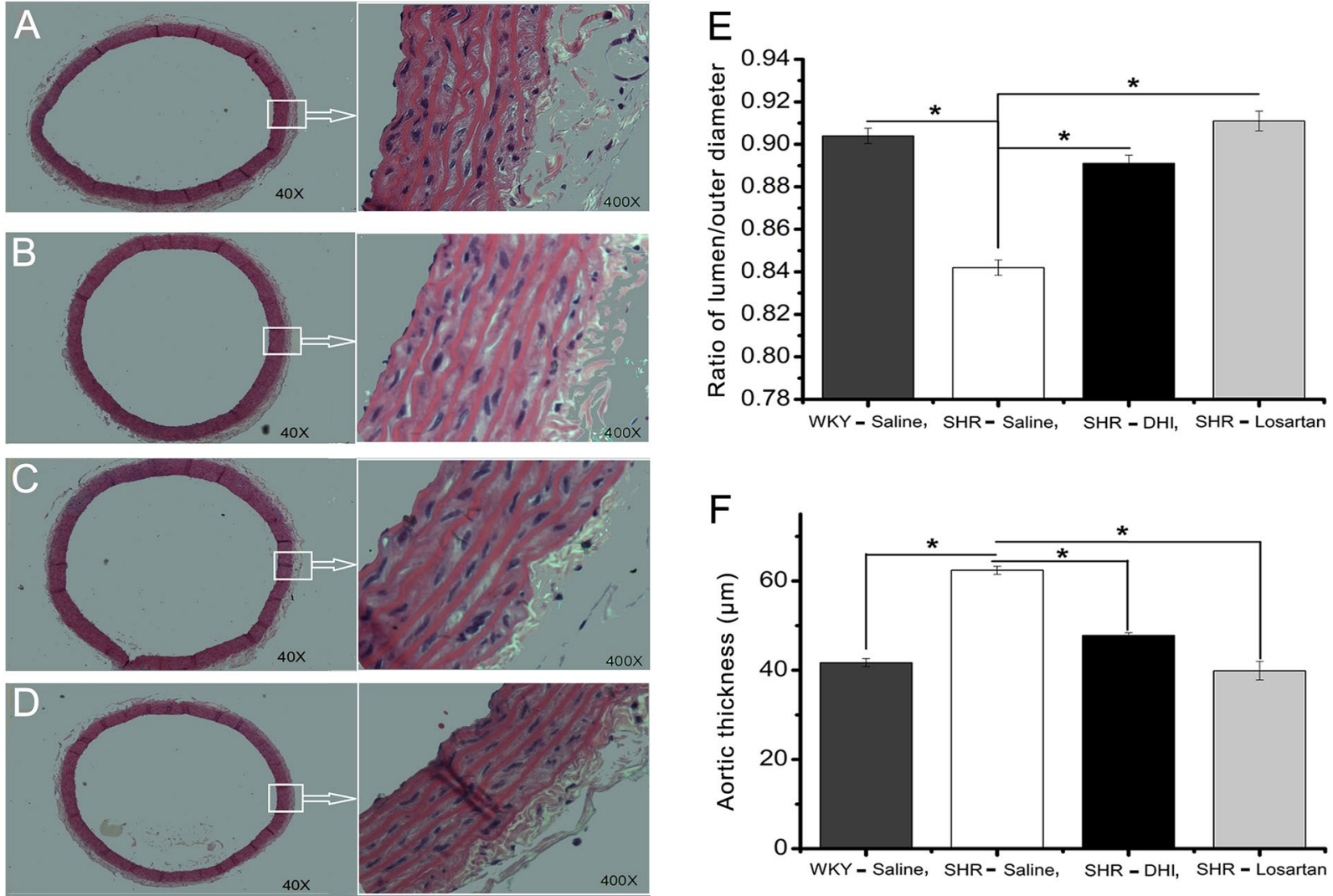

Figure 5. Effect of DHI on vascular remodeling in SHR. Histology of H\&E stain of thoracic aorta at $x 40$ and x400 (A-D) are shown. Summarized ratio of the inner to outer diameter of the thoracic aortas from the 4 groups of rats are quantified and presented in (E and F). (A) Saline treated WKY rats; B: Saline treated SHR rats; (C) SHR rats treated DHI; (D) SHR treated Losartan. Values represent mean $\pm \mathrm{SEM}$ ( $\mathrm{n}=4$ per group; $\mathrm{p}<0.01$ vs. SHR treated DHI).

The onset and development of hypertension is often accompanied by vascular structure and function abnormalities such as endothelial dysfunction and the hypertrophy, hyperplasia and connective tissue increase of the blood vessels ${ }^{55,56}$. In the current study, we found that DHI increased endothelium-dependent relaxation $(23 \pm 3 \%$, $\mathrm{p}<0.01$, Fig. 3C) but not endothelium-independent relaxation of aortas from SHR ( $>0.05$, Fig. 3D). This finding is consistent with our previous study using wild-type SD rats that DHI and one of its active components, Danshensu, promoted vasorelaxation in vivo (2 day-treatment) and ex vivo by a COX/PGI 2 -mediated pathway ${ }^{38}$. Interestingly, we have shown recently that a longer exposure (4 weeks) of DHI in SD rats further increased endothelium-dependent relaxation $(19 \% \pm 2 \%, \mathrm{p}<0.01$, data not shown) compared to the acute exposure $(2$ days) reported previously ${ }^{38}$. Consistent with these findings, overexpression of tissue kallikrein gene promoted KKS activation, which enhances prostacyclin 2 and nitric oxide biosynthesis, both of them facilitated the regulation of vascular responses ${ }^{18,57}$.

Additionally, increased vascular wall thickness is a common structural feature of hypertensive resistance vessels and conduit arteries such as the aorta ${ }^{22,58}$. Chronic hypertension changes the dimensions and properties of arterial wall and these alterations may affect arterial mechanics. Therefore, vascular remodeling, a pathological process involving VSMCs proliferation, migration, hypertrophy, vascular compliance reduction and narrowing of the vessels lumen, cause a major damage of hypertension ${ }^{59}$. Study of Bo Wang et al. ${ }^{22}$ demonstrated that Qin-Jiang-Ya-Tang (TCM) could reverse thoracic aortas remodeling in SHR. In our present study, DHI significantly decreased SHR aortic thickness compared with that of control group (Fig. 5E and F). Numerous studies have demonstrated that either tissue or serum kallikrein overexpression decreases vascular wall thickness, inhibited VSMCs proliferation and reversed vascular remodeling ${ }^{60,61}$, and our results indicated that the long-term effect of DHI on vascular remodeling could also be mediated by the KKS via kallikrein up-regulation.

Finally, it is worth noticing that the vast majority of over 200 differentially expressed genes by DHI treatment in the microarray study (supplemental material Table S2) remain unexplored. Future studies using combined approaches of bioinformatics and molecular biology may further elucidate DHI's molecular targets and therapeutic potentials in hypertensive resistance vessels. Also, to further validate the proposed anti-hypertensive mechanism of DHI via KKS modulation, future study need to focus on directions to show that blocking KKS by inhibitors or using gene knockout mice would prevent DHI mediated BP lowing, vasorelaxation and remodeling. Since we have only investigated the whole DHI, another required research direction is to define the precise active components that are responsible for the cardiovascular benefits as a complex herbal medicine. 

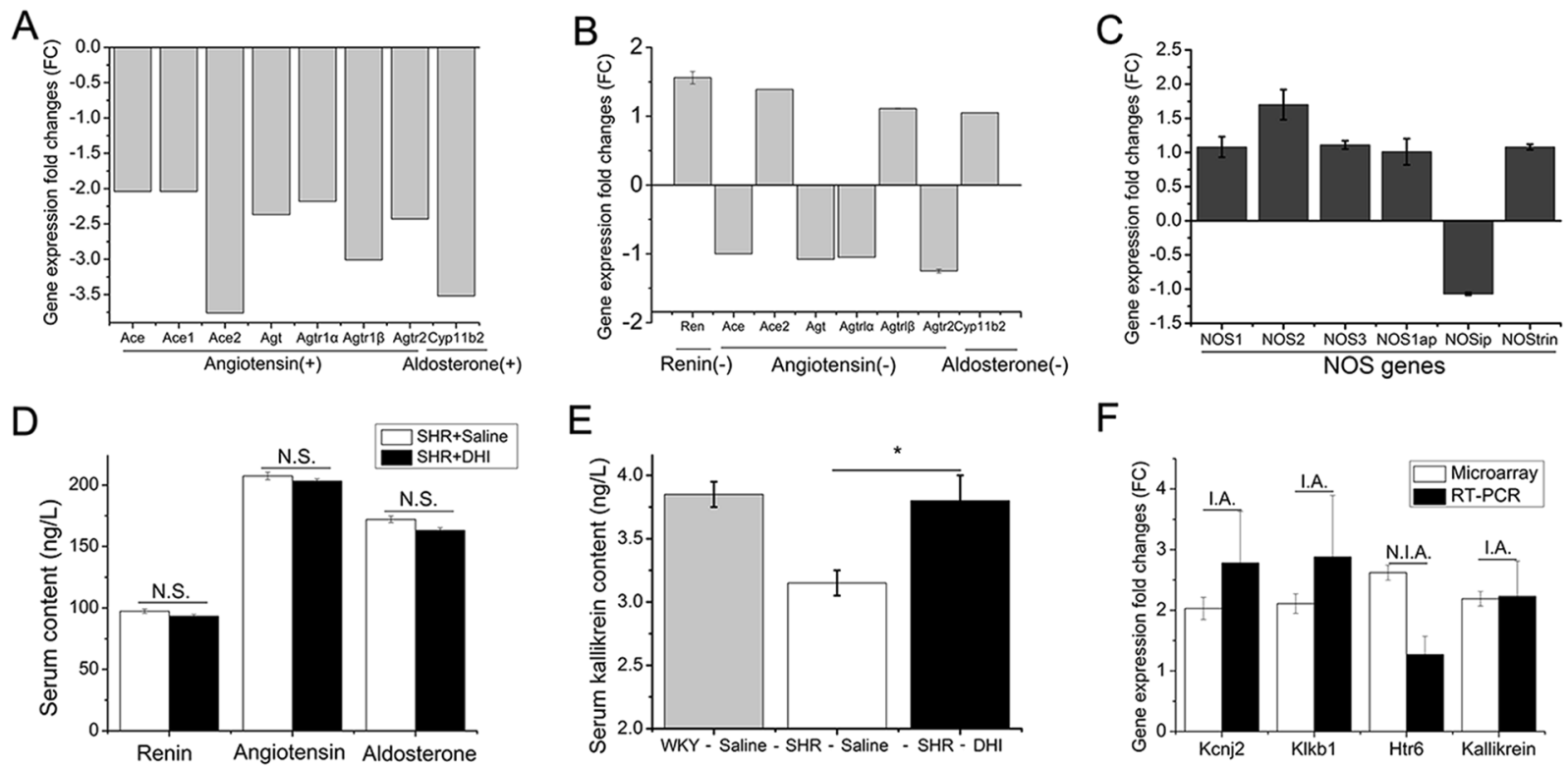

Figure 6. Gene expression changes by Microarray analysis and subsequent confirmation in SHR mesenteric vessels. (A) Microarray confirmation of down-regulation of RAAS genes by Losartan. (B and C) DHI did not alter RAAS and NOS genes in microarray profiling, respectively. (D) Serum renin, angiotensin and aldosterone contents were determined and no significant difference (N.S.) was detected between DHI treatment and the control groups. (E) Effect of DHI on serum kallikrein content determined by ELISA assay $\left({ }^{*} \mathrm{p}<0.05\right)$. (F) Other hypertension-associated genes that are differentially regulated by DHI in microarray and their confirmation by RT-PCR. RT-PCR gene expressions of Kcnj2, Klkb1, and kallikrein were in accordance (I.A) with microarray data whereas Htr6 gene expression was not in accordance (N.I.A.) with that obtained by microarrays. All data are expressed as mean $\pm \mathrm{SEM}, \mathrm{n}=4$.

In conclusion, Chinese medicine DHI effectively lowers blood pressure without causing significant adverse effect. This effect is attributable to DHI's ability of enhanced vasorelaxation and reduced vascular remodeling. The antihypertensive action and vascular protection by DHI in SHR model are at least in part mediated by KKS via up-regulation of kallikrein gene expression.

\section{Materials and Methods}

Reagents. DHI was supplied by HEZE BUCHANG PHARMACEUTICAL CO., LTD. Losartan was purchased from the Yangtze River Pharmaceutical Group Sichuan sea pureed pharmaceutical Co., LTD (Dujiangyan, Sichuan, China). Saline was purchased from China Otsuka pharmaceutical co., LTD (Tianjin, China). Adrenaline (AD) was purchased from (Shanghai Harvest Pharmaceutical Co., Ltd, China). All other reagents were purchased from Sigma-Aldrich (St Louis, MO). Losartan was dissolved and diluted by saline. In vivo/vitro DHI doses were according to our previous report ${ }^{38}$.

Animals and Treatment. Adult (12 week-old) male SHR and age-matched male normotensive Wistar-Kyoto (WKY) rats, weighting about $200 \mathrm{~g}$, were purchased from Vital River Experimental Animal Technology Co., LTD (Beijing, China). Rats were intraperitoneally injected with DHI $(2.5 \mathrm{~mL} / \mathrm{kg} /$ day $)$, Losartan $(20 \mathrm{mg} / \mathrm{kg} / \mathrm{day})$, and/ or saline $(2.5 \mathrm{~mL} / \mathrm{kg} /$ day $)$ for 4 weeks. They were maintained in a temperature-controlled room $\left(25^{\circ} \mathrm{C} \pm 1\right)$ under a cycle of 12 hours of light (beginning at 9:00 A.M.). All rats were given water and fed with standard chow. All animal care and operation procedures were in strict accord with the China Laboratory Animal Use Regulations and were approved by the Institutional Animal Care and Use Committee at Tianjin International Joint Academy of Biotechnology and Medicine (TJAB-JY-2011-002), Tianjin, China.

After 4 weeks of treatment, animals were anesthetized using intraperitoneal injection of $10 \%$ Chloralic Hydras. Serum from blood was collected post-anesthesia and stored at $-20^{\circ} \mathrm{C}$ for ELISA assay. Thoracic aortas and mesenteric micro-vessels were harvested. Aortas were placed in $10 \%$ formalin for hematoxylin and eosin (H\&E) staining, and mesenteric micro-vessels were frozen in liquid nitrogen for microarray analysis and real time RT-PCR verification.

BP Measurements. Conscious noninvasive tail-cuff BP measurements were performed every two days for 4 weeks after the initiation of the drug treatments using an 8-channel CODA noninvasive BP acquisition system (Kent Scientific Corporation, CT, USA) following the manufacturer's protocol. Measurements were recorded after 7 days of training. Systolic, diastolic and mean BP as well as heart rate was recorded. For BP recording, eight separate measurements were obtained and averaged for each rat.

Invasive Blood pressure and heart rate where measured using ADInstruments PowerLab 8/30 connected to bridge Amp, ML 221, invasive blood pressure (BP) was recorded alongside heart rate after rats were treated with DHI and changes in BP was observed. Briefly, Animals were anesthetized with urethane and surgical 
manipulation was carried out on the animals. A heparinized saline, $100 \mathrm{IU} / \mathrm{mL}$ was filled in the transducer in clean polyethylene catheter cannulated to the ventricle through the left common carotid artery to prevent blood clotting. BP and HR were continuously recorded for 3 min each as waveform curve and the software calculated their values.

Aortic Ring Assay. The aortic ring assay was performed as previous described ${ }^{62}$. Briefly, segments of aortas $(3 \mathrm{~mm})$ were dissected out and placed in organ baths containing $20 \mathrm{~mL}$ Krebs buffer (mM: $\mathrm{NaCl} \mathrm{118,} \mathrm{KCl}$ 4.7, $\mathrm{MgSO}_{4} 1.2, \mathrm{KH}_{2} \mathrm{PO}_{4} 1.2, \mathrm{NaHCO}_{3} 25, \mathrm{CaCl}_{2}$ 2.5, glucose 5.5); optical resting tension was determined in baseline experiments performed before treatment with the compounds under investigation. The rings were gradually stretched to an optimum preload of $2.0 \mathrm{~g}$ of force, determined in previous experiments in this laboratory ${ }^{38}$. Vessels were sub-maximally pre-contracted with $\mathrm{KCl}(60 \mathrm{mM})$, the endothelial function was evaluated by vascular relaxation in response to acetylcholine (Ach) and the vascular smooth muscle function was evaluated by vascular relaxation in response to sodium nitroprusside (SNP). Isometric forces were recorded with force transducers connected to a PowerLab/870 Eight-channel $100 \mathrm{kHz}$ A/D converter (AD Instruments, Sydney, Australia).

Isolation and Mounting of Small Mesenteric Artery Segments. Rats were killed by an overdose of isoflurane and the mesentery was removed and placed in cold Krebs-Ringer buffer (KRB) with the following composition (in $\mathrm{mM}$ ): $118.5 \mathrm{NaCl}, 4.7 \mathrm{KCl}, 2.5 \mathrm{CaCl}$, 1.2 MgSO4, 1.2 KH2PO4, 25.0 NaHCO3, and 5.5 D-glucose. From each rat, segments $(2 \mathrm{~mm})$ of the second-order branches of the superior mesenteric artery (MA) was carefully dissected and mounted on a wire-myograph (model 620 M; Danish Myotechnology, Aarhus, Denmark) for the recording of isometric force development. MAs were incubated for $30 \mathrm{~min}$ in KRB with continuous aeration in $95 \% \mathrm{O}_{2} / 5 \% \mathrm{CO}_{2}$ and were maintained at $37^{\circ} \mathrm{C}$. MAs were passively stretched according to a procedure first described by Halpern and Mulvany ${ }^{63}$. In brief, MAs were stretched stepwise to a passive wall tension of $90 \%$ of the internal circumference achieved when they were exposed to a passive tension yielding a transmural pressure of $100 \mathrm{mmHg}$. At this passive wall tension, MAs were contracted with high $\mathrm{K}^{+} \mathrm{KRB}(60 \mathrm{mmol} / \mathrm{L} \mathrm{KCl}$ in $\mathrm{KRB}$ solution; replacing equimolar $\mathrm{NaCl}$ with $\mathrm{KCl}$ ), thus generating a stable contraction that reached a plateau after $10-15 \mathrm{~min}$. This active wall tension was set to a $100 \%$ contraction level.

RNA Extraction. Total RNAs were extracted from rat mesenteric micro-vessels using RNeasy Mini Kit (QIAGEN, Valencia, CA), following the manufacturer's protocol. RNA concentrations were measured by ultraviolet spectrophotometer and denaturing gel electrophoresis. All raw RNA samples were purified and concentration adjusted to $50 \mathrm{ng} / \mu \mathrm{L}$. The RNA samples were stored in liquid nitrogen before further processing for microarray analysis and real time RT-PCR verification.

Microarray Analysis. Only the RNAs with RNA integrity numbers (RINs) greater than 7.0 and a 28SrRNA/18SrRNA ratio more than 0.7 were used for microarray experiments. Gene expression data were generated using Affymetrix Rat 230 plus 2.0 arrays (Affymetrix, Inc. USA) for 4 independent animals. Each array consists of 31,000 probe sets/genes (Seen detailed procedures for Supplemental Materials).

ELISA Analysis. Serum from each treatment group was collected and the contents of renin, angiotensin, aldosterone and kallikrein were detected using ELISA Kit (R\&D Systems, Inc. MN USA) according to the manufacturer's instruction (Seen detailed procedures for Supplemental Materials).

Histological Studies. Aorta specimens from each animal were fixed in 10\% formalin, paraffin-embedded and sectioned at $5 \mu \mathrm{m}$ thickness, deparaffinized and rehydrated. Histological staining was performed as previous described $^{64}$. H\&E were used to evaluate the general histology of aorta. The whole aortas with lumen and outer diameters were photographed and the mean arterial wall thickness (defined as the ratio of lumen/outer diameter) was determined by a computerized ImageJ software (National Institutes of Health, USA).

Statistical Analysis. All values are presented as means \pm SEM with "n" being the number of individual rats. Statistical analyses were performed by one-way ANOVA followed by Bonferroni multiple comparisons test (95\% confidence interval) from SPSS11.5 and we used Origin 8.5.1 software (Origin Lab Ltd, USA) for data analyses. Values of $\mathrm{p}<0.05$ were considered to be statistically significant.

\section{References}

1. He, J. et al. Premature deaths attributable to blood pressure in China: a prospective cohort study. Lancet 374, 1765-1772 (2009).

2. Lewington, S. et al. The Burden of Hypertension and Associated Risk for Cardiovascular Mortality in China. JAMA internal medicine 176, 524-532 (2016).

3. Oladapo, O. O. et al. Target-organ damage and cardiovascular complications in hypertensive Nigerian Yoruba adults: a crosssectional study. Cardiovascular journal of Africa 23, 379-384 (2012).

4. Nadar, S. K., Tayebjee, M. H., Messerli, F. \& Lip, G. Y. Target organ damage in hypertension: pathophysiology and implications for drug therapy. Current pharmaceutical design 12, 1581-1592 (2006).

5. Battegay, E. J., de Miguel, L. S., Petrimpol, M. \& Humar, R. Effects of anti-hypertensive drugs on vessel rarefaction. Current opinion in pharmacology 7, 151-157 (2007).

6. Draaijer, P., Le Noble, J. L., Leunissen, K. M. \& Struyker-Boudier, H. A. The microcirculation and essential hypertension. The Netherlands journal of medicine 39, 158-169 (1991).

7. Fukuda, S., Yasu, T., Kobayashi, N., Ikeda, N. \& Schmid-Schonbein, G. W. Contribution of fluid shear response in leukocytes to hemodynamic resistance in the spontaneously hypertensive rat. Circulation research 95, 100-108 (2004)

8. Xiong, X. et al. Chinese herbal formulas for treating hypertension in traditional Chinese medicine: perspective of modern science. Hypertension research: official journal of the Japanese Society of Hypertension 36, 570-579 (2013).

9. Tang, Y. et al. Cardiovascular protection with danshensu in spontaneously hypertensive rats. Biological \& pharmaceutical bulletin 34, 1596-1601 (2011). 
10. Yoshida, H. \& Ura, N. [Role of kallikrein-kinin system in blood pressure regulation]. Nihon rinsho. Japanese journal of clinical medicine 64(Suppl 5), 89-93 (2006).

11. Chao, J., Bledsoe, G., Yin, H. \& Chao, L. The tissue kallikrein-kinin system protects against cardiovascular and renal diseases and ischemic stroke independently of blood pressure reduction. Biological chemistry 387, 665-675 (2006).

12. Rhaleb, N. E., Yang, X. P., Nanba, M., Shesely, E. G. \& Carretero, O. A. Effect of Chronic Blockade of the Kallikrein-Kinin System on the Development of Hypertension in Rats. Hypertension 37, 121-128 (2001).

13. Griol-Charhbili, V. et al. Tissue kallikrein deficiency and renovascular hypertension in the mouse. American journal of physiology. Regulatory, integrative and comparative physiology 296, R1385-1391 (2009).

14. Potier, L. et al. Tissue kallikrein, blood pressure regulation, and hypertension: insight from genetic kallikrein deficiency. Biological chemistry 394, 329-333 (2013).

15. Yu, H. Z., Xie, L. D., Zhu, P. L., Xu, C. S. \& Wang, H. J. Human tissue kallikrein 1 gene delivery inhibits PDGF-BB-induced vascular smooth muscle cells proliferation and upregulates the expressions of p27Kip1 and p2lCip1. Molecular and cellular biochemistry 360, 363-371 (2012).

16. Zhu, P. L. et al. [Effects of human tissue kallikrein gene delivery on the proliferation of vascular smooth muscle cells]. Zhonghua xin xue guan bing za zhi 38, 739-744 (2010).

17. Yu, H. Z., Xie, L. D., Zhu, P. L. \& Xu, C. S. [Effects of human tissue kallikrein gene transfer on the migration of vascular smooth muscule cells]. Nan fang yi ke da xue xue bao=Journal of Southern Medical University 30, 746-749 (2010).

18. Abdallah, R. T. et al. Plasma kallikrein promotes epidermal growth factor receptor transactivation and signaling in vascular smooth muscle through direct activation of protease-activated receptors. The Journal of biological chemistry 285, 35206-35215 (2010).

19. Stone, O. A. et al. Critical role of tissue kallikrein in vessel formation and maturation: implications for therapeutic revascularization. Arteriosclerosis, thrombosis, and vascular biology 29, 657-664 (2009).

20. de la Sierra, A. \& Barrios, V. Blood pressure control with angiotensin receptor blocker-based three-drug combinations: key trials. Advances in therapy 29, 401-415 (2012).

21. Hou, S.-z. et al. Effect of the flavonoid fraction of Lithocarpus polystachyus Rehd. on spontaneously hypertensive and normotensive rats. Journal of ethnopharmacology 143, 441-447 (2012).

22. Wang, B. et al. Effect of traditional Chinese medicine Qin-Dan-Jiang-Ya-Tang on remodeled vascular phenotype and osteopontin in spontaneous hypertensive rats. Journal of ethnopharmacology 110, 176-182 (2007).

23. Wen, Z. et al. Discovery of molecular mechanisms of traditional Chinese medicinal formula Si-Wu-Tang using gene expression microarray and connectivity map. PloS one 6, e18278 (2011)

24. Chavan, P., Joshi, K. \& Patwardhan, B. DNA microarrays in herbal drug research. Evidence-based complementary and alternative medicine: eCAM 3, 447-457 (2006).

25. Zhang, J. et al. Microarray expression profiling of Yersinia pestis in response to berberine. Planta medica 75, 396-398 (2009).

26. Liu, X., Wu, Z., Yang, K., Ding, H. \& Wu, Y. Quantitative analysis combined with chromatographic fingerprint for comprehensive evaluation of Danhong injection using HPLC-DAD. Journal of pharmaceutical and biomedical analysis 76, 70-74 (2013).

27. Fu, T. T., Wang, C. J., Min, C. Y. \& Huang, X. H. [Effects of danhong injection on experimental atherosclerosis rabbit model and its mechanism]. Zhong yao cai=Zhongyaocai $=$ Journal of Chinese medicinal materials 32, 1720-1722 (2009).

28. Liu, H. T. et al. Characterization of in vivo antioxidant constituents and dual-standard quality assessment of Danhong injection. Biomedical chromatography: BMC (2012).

29. Jiang, M. et al. Quantitative profiling of polar metabolites in herbal medicine injections for multivariate statistical evaluation based on independence principal component analysis. PloS one 9, e105412 (2014).

30. ZHANG, Q.-Q. et al. Rapid separation and identification of multiple constituents in Danhong Injection by ultra-high performance liquid chromatography coupled to electrospray ionization quadrupole time-of-flight tandem mass spectrometry. Chinese journal of natural medicines 14, 147-160 (2016).

31. Li, C. X., Tang, J. F., Meng, F. \& Li, X. L. [Security revaluation study based on nested case-control method of Danhong injection]. Zhongguo Zhong yao za zhi=Zhongguo zhongyao zazhi=China journal of Chinese materia medica 37, 2735-2738 (2012).

32. He, Y. et al. Protective effect of Danhong injection on cerebral ischemia-reperfusion injury in rats. Journal of ethnopharmacology 144, 387-394 (2012).

33. Zhao, P.X. \& Jiang, S. [Effect of danhong injection on ET-1, sP-sel, and hs-CRP in patients with acute coronary syndrome undergoing percutaneous coronary intervention]. Zhongguo Zhong xi yi jie he za zhi Zhongguo Zhongxiyi jiehe zazhi=Chinese journal of integrated traditional and Western medicine/Zhongguo Zhong xi yi jie he xue hui, Zhongguo Zhong yi yan jiu yuan zhu ban 31, 11-14 (2011).

34. Guan, Y. et al. Dissection of mechanisms of a chinese medicinal formula: danhong injection therapy for myocardial ischemia/ reperfusion injury in vivo and in vitro. Evidence-Based Complementary and Alternative Medicine 2013 (2013).

35. Mao, H. P. et al. Danhong injection attenuates isoproterenol-induced cardiac hypertrophy by regulating p38 and NF-kappab pathway. Journal of ethnopharmacology 186, 20-29 (2016).

36. Chen, J. et al. Amelioration of cardiac dysfunction and ventricular remodeling after myocardial infarction by danhong injection are critically contributed by anti-TGF- $\beta$-mediated fibrosis and angiogenesis mechanisms. Journal of ethnopharmacology 194, 559-570 (2016).

37. Su, X. et al. Vasorelaxant activities of Danhong injection and their differential effects on the rat abdominal aorta and mesenteric artery. Journal of cardiovascular pharmacology 65, 62-71 (2015).

38. Wang, D. et al. Vascular reactivity screen of Chinese medicine danhong injection identifies Danshensu as a NO-independent but PGI2-mediated relaxation factor. Journal of cardiovascular pharmacology 62, 457-465 (2013).

39. Vetriakov, O. V., Bykov, V. N., Iudin, M. A. \& Nikiforov, A. S. [Effect of losartan on acute renal failure induced by severe ethylene glycol poisoning in rats]. Eksperimental'naia i klinicheskaia farmakologiia 75, 21-24 (2012).

40. Sim, M. K. \& Singh, M. Decreased responsiveness of the aortae of hypertensive rats to acetylcholine, histamine and noradrenaline. British journal of pharmacology 90, 147-150 (1987).

41. Li, J. \& Bukoski, R. D. Endothelium-dependent relaxation of hypertensive resistance arteries is not impaired under all conditions. Circulation research 72, 290-296 (1993).

42. Fujii, K. et al. Decreased endothelium-dependent hyperpolarization to acetylcholine in smooth muscle of the mesenteric artery of spontaneously hypertensive rats. Circulation research 70, 660-669 (1992)

43. Liu, H. et al. Danhong inhibits oxidized low-density lipoprotein-induced immune maturation of dentritic cells via a peroxisome proliferator activated receptor gamma-mediated pathway. Journal of pharmacological sciences 119, 1-9 (2012).

44. Yao, J. Y., Zhi, M., Cao, W. T., Huang, Y. \& Li, C. J. Successful treatment with danhong injection for hepatic veno-occlusive disease. Hepato-gastroenterology 58, 992-995 (2011).

45. Wang, L. et al. Antihypertensive effects of olmesartan compared with other angiotensin receptor blockers: a meta-analysis. American journal of cardiovascular drugs: drugs, devices, and other interventions 12, 335-344 (2012).

46. Nie, P. H., Zhang, L., Zhang, W. H., Rong, W. F. \& Zhi, J. M. The effects of hydroxysafflor yellow A on blood pressure and cardiac function. Journal of ethnopharmacology 139, 746-750 (2012).

47. Kang, D. G., Yun, Y. G., Ryoo, J. H. \& Lee, H. S. Anti-hypertensive effect of water extract of danshen on renovascular hypertension through inhibition of the renin angiotensin system. The American journal of Chinese medicine 30, 87-93 (2002). 
48. Kim, D. D., Sanchez, F. A., Duran, R. G., Kanetaka, T. \& Duran, W. N. Endothelial nitric oxide synthase is a molecular vascular target for the Chinese herb Danshen in hypertension. American journal of physiology. Heart and circulatory physiology 292, H2131-2137 (2007).

49. Akazawa, H. \& Komuro, I. [Research of RAAS: progress and perspective]. Nihon rinsho. Japanese journal of clinical medicine 70, $1471-1476$ (2012).

50. Su, J. B. Different cross-talk sites between the renin-angiotensin and the kallikrein-kinin systems. Journal of the renin-angiotensinaldosterone system: JRAAS 15, 319-328 (2014).

51. Stoka, V. \& Turk, V. A structural network associated with the kallikrein-kinin and renin-angiotensin systems. Biological chemistry 391, 443-454 (2010).

52. Griol-Charhbili, V. et al. Tissue kallikrein deficiency and renovascular hypertension in the mouse. American Journal of Physiology Regulatory, Integrative and Comparative Physiology 296, R1385-R1391 (2009).

53. Waeckel, L. et al. Antihypertensive role of tissue kallikrein in hyperaldosteronism in the mouse. Endocrinology 153, 3886-3896 (2012).

54. Zhao, C. et al. Gene therapy with human tissue kallikrein reduces hypertension and hyperinsulinemia in fructose-induced hypertensive rats. Hypertension 42, 1026-1033 (2003).

55. Greene, A. S., Tonellato, P. J., Lui, J., Lombard, J. H. \& Cowley, A. W. Microvascular rarefaction and tissue vascular resistance in hypertension. American Journal of Physiology - Heart and Circulatory Physiology 256, H126-H131 (1989).

56. Rizzoni, D. et al. Vascular structural and functional alterations before and after the development of hypertension in SHR. American journal of hypertension 7, 193-200 (1994).

57. Chao, J. et al. Tissue kallikrein in cardiovascular, cerebrovascular and renal diseases and skin wound healing. Biological chemistry 391, 345-355 (2010).

58. Chamiot-Clerc, P., Renaud, J. F. \& Safar, M. E. Pulse pressure, aortic reactivity, and endothelium dysfunction in old hypertensive rats. Hypertension 37, 313-321 (2001).

59. Hanze, J., Weissmann, N., Grimminger, F., Seeger, W. \& Rose, F. Cellular and molecular mechanisms of hypoxia-inducible factor driven vascular remodeling. Thrombosis and haemostasis 97, 774-787 (2007).

60. Martins-Oliveira, A. et al. Contrasting effects of aliskiren versus losartan on hypertensive vascular remodeling. Int J Cardiol 167, 1199-1205 (2013).

61. Miyata, K. et al. Possible involvement of Rho-kinase in aldosterone-induced vascular smooth muscle cell remodeling. Hypertension research: official journal of the Japanese Society of Hypertension 31, 1407-1413 (2008).

62. Zhu, Y. et al. Abnormal vascular function and hypertension in mice deficient in estrogen receptor beta. Science 295, 505-508 (2002).

63. Halpern, W. \& Mulvany, M. J. Tension responses to small length changes of vascular smooth muscle cells [proceedings]. J Physiol 265, 21P-23P (1977).

64. Jing, L. et al. Inhibition of extracellular signal-regulated kinases ameliorates hypertension-induced renal vascular remodeling in rat models. International journal of molecular sciences 12, 8333-8346 (2011).

\section{Acknowledgements}

We thank the members of our laboratory, particularly Dr. Yantong Xu, Xiaoxuan Tian, Yuefei Wang and Guixiang Pan, for stimulating discussions; Miao Wang and Qi Zhang for assistance in some of the vascular ring experiments; Shuang He, Tiechan Zhao, and Rongrong Chen for taking part in sample preparations. YZ certifies that (1) all persons who have made substantial contributions to the manuscript, including data collection and analysis, writing and editing assistance who do not fulfill authorship criteria, are named with their specific contributions here; (2) all persons named in this section have provided the corresponding author with written permission to be named in the manuscript. This work was supported by grants from the Program for Changjiang Scholars and Innovative Research Team in University (PCSIRT No. IRT-14R41), National Major New Drug Discovery (2013ZX09201020) and the International Cooperation Project of MOST, China (2013DFA31620).

\section{Author Contributions}

Y.Z. conceived and designed the study; X.Y., D.W., and J.H. performed experiments; X.Y. and Y.Z. wrote the manuscript; J.O., G.F., J.H., G.Q., and X.G. reviewed and edited the manuscript.

\section{Additional Information}

Supplementary information accompanies this paper at doi:10.1038/s41598-017-04661-1

Competing Interests: The authors declare that they have no competing interests.

Publisher's note: Springer Nature remains neutral with regard to jurisdictional claims in published maps and institutional affiliations.

Open Access This article is licensed under a Creative Commons Attribution 4.0 International

License, which permits use, sharing, adaptation, distribution and reproduction in any medium or format, as long as you give appropriate credit to the original author(s) and the source, provide a link to the Creative Commons license, and indicate if changes were made. The images or other third party material in this article are included in the article's Creative Commons license, unless indicated otherwise in a credit line to the material. If material is not included in the article's Creative Commons license and your intended use is not permitted by statutory regulation or exceeds the permitted use, you will need to obtain permission directly from the copyright holder. To view a copy of this license, visit http://creativecommons.org/licenses/by/4.0/.

(c) The Author(s) 2017 